\title{
Parametric Factorizations of Second-, Third- and Fourth-Order Linear Partial Differential Operators with a Completely Factorable Symbol on the Plane
}

\author{
Ekaterina Shemyakova
}

\begin{abstract}
Parametric factorizations of linear partial operators on the plane are considered for operators of orders two, three and four. The operators are assumed to have a completely factorable symbol. It is proved that "irreducible" parametric factorizations may exist only for a few certain types of factorizations. Examples are given of the parametric families for each of the possible types. For the operators of orders two and three, it is shown that any factorization family is parameterized by a single univariate function (which can be a constant function).
\end{abstract}

Mathematics Subject Classification (2000). Primary 47F05; Secondary 68W30. Keywords. partial differential, LPDO, factorization, parametric factorization, family of factorizations.

\section{Introduction}

The factorization of the Linear Partial Differential Operator (LPDO)

$$
L=\sum_{i_{1}+\cdots+i_{n} \leq d} a_{i_{1} \ldots i_{n}} D_{1}^{i_{1}} \ldots D_{n}^{i_{n}}
$$

where the coefficients belong to some differential ring, is an important technique, used by modern algorithms for the integration of the corresponding Linear Partial Differential Equation (LPDE) $L(f)=0$. Many of the algorithms have appeared as advanced modifications and generalizations of the well-known Laplace-Euler

This work was supported by Austrian Science Foundation (FWF) under the project SFB F013/F1304. 
transformation method (the Laplace cascade method) which serves as a method for computing a general solution for the bivariate second-order linear hyperbolic equations

$$
u_{x y}+a u_{x}+b u_{y}+c=0, \quad a=a(x, y), b=b(x, y), c=c(x, y) .
$$

The basis of the algorithm is the fact that whenever the operator in (1.1) factors, for instance,

$$
L=D_{x} \circ D_{y}+a D_{x}+b D_{y}+c=\left(D_{x}+b\right) \circ\left(D_{y}+a\right),
$$

the problem of determining all the integrals of the equation (1.1) reduces to the problem of integrating the two first-order equations

$$
\begin{aligned}
\left(D_{x}+b\right)\left(u_{1}\right) & =0, \\
\left(D_{y}+a\right)(u) & =u_{1} .
\end{aligned}
$$

Accordingly, one obtains the general solution of the original equation (1.1) as

$$
z=\left(p(x)+\int q(y) e^{\int a d y-b d x} d y\right) e^{-\int a d y}
$$

with two arbitrary functions $p(x)$ and $q(y)$. If it is the case that the operator (1.2) is not factorable, one may construct (by Laplace transformations) the sequence of operators

$$
\ldots \leftrightarrow L_{-2} \leftrightarrow L_{-1} \leftrightarrow L \leftrightarrow L_{1} \leftrightarrow L_{2} \leftrightarrow \ldots,
$$

such that the kernel of any descendant can be found from the kernel of the initial operator $L$. Thus, whenever one of those operators becomes factorable, the general solution of the initial problem can be found.

Since the 18th century, many generalizations of the method have been given (for example 1, 2, 3, 4, 7, 8, 9, 10, 11, 12, 15, 19), and the problem of constructing factorization algorithms for different kinds of differential operators has become important. Over the last decade, a number of new modifications of the classical algorithms for the factorization of LPDOs (for example, [5, 6, 13, 14, 17, 18, ) have been given. So far, most of the activity has addressed the hyperbolic case, and there is as yet a lack of knowledge concerning the non-hyperbolic case.

There is a distinction in kind between the two cases. A factorization of a hyperbolic LPDO on the plane is determined uniquely by a factorization of the operator's symbol (principal symbol) (see Theorem 2.5 and [5]). Thus, the operator (1.2) may have at most one factorization of each of the forms $\left(D_{x}+\ldots\right) \circ\left(D_{y}+\ldots\right)$ and $\left(D_{y}+\ldots\right) \circ\left(D_{x}+\ldots\right)$. On the other hand for the non-hyperbolic operator $D_{x x}$ there is the stereotypical example

$$
D_{x x}=D_{x} \circ D_{x}=\left(D_{x}+\frac{1}{x+c}\right) \circ\left(D_{x}-\frac{1}{x+c}\right),
$$

where $c$ is an arbitrary parameter. A more significant example is provided by the Landau operator $L=D_{x x}\left(D_{x}+x D_{y}\right)+2 D_{x x}+2(x+1) D_{x y}+D_{x}+(x+1) D_{y}$, 
which factors as

$$
L=\left(D_{x}+1+\frac{1}{x+c(y)}\right) \circ\left(D_{x}+1-\frac{1}{x+c(y)}\right) \circ\left(D_{x}+x D_{y}\right),
$$

where the function $c(y)$ is arbitrary. This shows that some LPDOs may have essentially different factorizations, and, further, that the factors may contain arbitrary parameters or even functions. Thus we may have families of factorizations.

An LPDO is hyperbolic if its symbol is completely factorable (all factors are of first order) and each factor has multiplicity one. In the present paper we consider the case of LPDOs that have completely factorable symbols, without any additional requirement. We prove that "irreducible" (see Definition 2.3) families of factorizations can exist only for a few certain types of factorizations. For these cases explicit examples are given. For operators of orders two and three, it is shown that a family may be parameterized by at most one function in one variable. Our investigations cover the case of ordinary differential operators as well. Some related remarks about parametric factorizations for ordinary differential operators may be found in [16.

The paper is organized as follows. In section 2, we give some general definitions and remarks about factorizations and families of factorizations of LPDOs. Then in section 3, we describe our basic tool - local linearization. In sections 4, 5 , and 6 we formulate and prove results about LPDOs of orders two, three, and four respectively. The last section contains conclusions, and some ideas for future work.

\section{Preliminaries}

We consider a field $K$ with a set $\Delta=\left\{\partial_{1}, \ldots, \partial_{n}\right\}$ of commuting derivations acting on it. We work with the ring of linear differential operators $K[D]=K\left[D_{1}, \ldots, D_{n}\right]$, where $D_{1}, \ldots, D_{n}$ correspond to the derivations $\partial_{1}, \ldots, \partial_{n}$, respectively. Any operator $L \in K[D]$ is of the form

$$
L=\sum_{|J| \leq d} a_{J} D^{J},
$$

where $a_{J} \in K, J \in \mathbf{N}^{n}$ and $|J|$ is the sum of the components of $J$. The symbol of $L$ is the homogeneous polynomial $\operatorname{Sym}_{L}=\sum_{|J|=d} a_{J} X^{J}$. The operator $L$ is hyperbolic if the polynomial $\mathrm{Sym}_{L}$ has exactly $d$ different factors.

Definition 2.1. Let $L, F_{1}, \ldots, F_{k} \in K[D]$. A factorization $L=F_{1} \circ \cdots \circ F_{k}$ is said to be of the factorization type $\left(S_{1}\right) \ldots\left(S_{k}\right)$, where $S_{i}=\operatorname{Sym}_{F_{i}}$ for all $i$.

Definition 2.2. Let $L \in K[D]$. We say that

$$
L=F_{1}(T) \circ \cdots \circ F_{k}(T)
$$

is a family of factorizations of $L$ parameterized by the parameter $T$ if, for any value $T=T_{0}$, we have that $F_{1}\left(T_{0}\right), \ldots, F_{k}\left(T_{0}\right)$ are in $K[D]$ and $L=F_{1}\left(T_{0}\right) \circ \cdots \circ F_{k}\left(T_{0}\right)$ 
holds. Here $T$ is an element from the space of parameters $\mathbb{T}$. Usually $\mathbb{T}$ is the Cartesian product of some (functions') fields, in which the number of variables is less than that in $K$.

We often consider families without mentioning or designating the corresponding operator; we define the symbol and the order of the family to be equal to symbol and order of the operator.

Definition 2.3. We say that a family of factorizations (2.2) is reducible, if there is $i, 1 \leq i \leq k$, such that the product

$$
F_{1}(T) \circ \cdots \circ F_{i}(T)
$$

does not depend on the parameter $T$ (in this case the product $F_{i+1}(T) \circ \cdots \circ F_{k}(T)$ does not depend on the parameters as well). Otherwise the family is reducible.

Thus, the family (1.3) is reducible. However, the product of the first two factors does not depend on the parameter, while the factors themselves do. So we have an example of a second-order irreducible family of factorizations.

Remark 2.4. Note that any irreducible family of the type $\left(S_{1}\right)\left(S_{2}\right)\left(S_{3}\right)$ serves as an irreducible family of the types $\left(S_{1} S_{2}\right)\left(S_{3}\right)$ and $\left(S_{1}\right)\left(S_{2} S_{3}\right)$ as well. Indeed, the irreducibility of the family of the type $\left(S_{1}\right)\left(S_{2}\right)\left(S_{3}\right)$ means that the product of the first and the second factors, as well as that of the second one and the third one, depends on the parameter.

Analogous property enjoys the families of arbitrary orders.

Theorem 2.5. 5. Let $L \in K[D]$, and $\operatorname{Sym}_{L}=S_{1} \ldots S_{k}$, and let the $S_{i}$ be pairwise coprime. There is at most one factorization of $L$ of the type $\left(S_{1}\right) \ldots\left(S_{k}\right)$.

The theorem implies that, for instance, there are no irreducible families of the types $(X)\left(Y^{3}\right)$ or $\left(X^{2}\right)\left(Y^{2}\right)$.

Remark 2.6. The properties of factorizations, such as the existence of the factorizations, or the number of parameters, or again the number of variables in parametric functions, are invariant under a change of variables and the gauge transformations $L \mapsto g^{-1} L g, g \in K$, of the initial operator.

Definition 2.7. We say that a partial differential operator $L \in K[D]$ is almost ordinary if it is an ordinary differential operator in some system of coordinates (transformation's functions belong to $K$ ).

\section{The Linearized Problem}

The basic tool in our study of families of factorizations will be their linearization. Let an operator $L \in K[D]$ have a family of factorizations

$$
L=M_{1}(T) \circ M_{2}(T),
$$

parameterized by some parameters $T=\left(t_{1}, \ldots, t_{k}\right)$, with $M_{1}(T), M_{2}(T) \in K[D]$. By means of a multiplication by a function from $K$, one can make the symbols 
of $M_{1}(T)$ and $M_{2}(T)$ independent of the parameters. Take some point $T_{0}$ as an initial point, make the substitution $T \rightarrow T_{0}+\varepsilon R$, and equates the coefficient at the power $\varepsilon$. This implies

$$
F_{1} \circ L_{2}+L_{1} \circ F_{2}=0 .
$$

where we have denoted the initial factorization factors by $L_{i}=M_{i}\left(T_{0}\right)$, and $F_{i}=$ $F_{i}(R)$ for $i=1,2$. Analogously, for factorizations into three factors we get

$$
F_{1} \circ L_{2} \circ L_{3}+L_{1} \circ F_{2} \circ L_{3}+L_{1} \circ L_{2} \circ F_{3}=0 \text {. }
$$

In the paper we apply the linearization to obtain some important information about families of factorizations.

\section{Second-Order Operators}

Theorem 4.1. A second-order operator in $K\left[D_{x}, D_{y}\right]$ has a family of factorizations (in some extension of the field $K$ ) if and only if it is almost ordinary. Any such family is unique for a given operator. Further, in appropriate variables it has the form

$$
\left(D_{x}+a+\frac{Q}{W+f_{1}(y)}\right) \circ\left(D_{x}+b-\frac{Q}{W+f_{1}(y)}\right),
$$

where $Q=e^{\int(b-a) d x}, W=\int Q d x, a, b \in K$, and $f_{1}(y) \in K$ is a parameter.

Proof. Consider a second-order operator $L \in K\left[D_{x}, D_{y}\right]$. By a change of variables we can make the symbol of $L$ equal to either $X^{2}$ or $X Y$. In the latter case, $L$ has no family of factorizations because of Theorem 2.5 .

Consider the case $\operatorname{Sym}_{L}=X^{2}$. Then operator $L$ has a factorization only if it is ordinary. Suppose we know one factorization: $L=L_{1} \circ L_{2}=\left(D_{x}+a\right) \circ\left(D_{x}+b\right)$, where $a, b \in K$, and we are interested in deciding whether there exists a family. Consider the linearized problem, that is the equation (3.1) w.r.t. $F_{1}, F_{2} \in K$ : $F_{1} \circ L_{2}+L_{1} \circ F_{2}=0$. The equation always has a solution

$$
\left\{\begin{array}{l}
F_{1}=f_{1}(y) e^{(b-a) x} \\
F_{2}=-F_{1}
\end{array}\right.
$$

where $f_{1}(y) \in K$ is a parameter function. Thus, any family can be parameterized by only one function of one variable.

In fact, such a family always exists, and it is given explicitly in the statement of the theorem. Moreover, one can prove straightforwardly that such a family is unique for a given operator $L$.

\section{Third-Order Operators}

Theorem 5.1. Let a third-order operator in $K\left[D_{x}, D_{y}\right]$ with the completely factorable symbol has an irreducible family of factorizations. Then it is almost ordinary. 
Any such family depends by at most three (two) parameters if the number of factors in factorizations is three (two). Each of these parameters is a function of one variable.

Proof. Consider a third-order operator $L$ in $K\left[D_{x}, D_{y}\right]$. For the symbol $\mathrm{Sym}_{L}$ only the following three are possible: it has exactly three, two, or no coprime factors. In the first case no family is possible because of Theorem 2.5.

Suppose exactly two factors of the symbol are coprime. Thus, in some variables the symbol of $L$ is $X^{2} Y$. Consider factorization into two factors. Then the following types of factorizations are possible: $(X)(X Y),(Y)\left(X^{2}\right),(X Y)(X),\left(X^{2}\right)(Y)$. By Theorem 2.5. there is no family of factorizations of the types $(Y)\left(X^{2}\right),\left(X^{2}\right)(Y)$. Because of the symmetry, it is enough to consider just the case $(X)(X Y)$. Indeed, if there exists a family of the type $(X Y)(X)$ for some operator $L$ of the general form (2.1). Then the adjoint operator

$$
L^{t}(f)=\sum_{|J| \leq d}(-1)^{|J|} D^{J}\left(a_{J} f\right) .
$$

has a family of the type $(X)(X Y)$, and the number of parameters in the family is the same.

Thus, we consider a factorization of the factorization type $(X)(X Y)$ :

$$
L=L_{1} \circ L_{2}=\left(D_{x}+r\right) \circ\left(D_{x y}+a D_{x}+b D_{y}+c\right),
$$

where $r, a, b, c \in K$ as the initial factorization for some family of factorizations of the factorization type $(X)(X Y)$. By means of the gauge transformations, we make the coefficient $a$ equal zero in this initial factorization (of course, the coefficient at $D_{x}$ in the second factor of other factorizations of the family may be still nonzero). To study possible families in this case, we consider the linearized problem: the equation $F_{1} \circ L_{2}+L_{1} \circ F_{2}=0$ w.r.t. $F_{1}=r_{1}, F_{2}=a_{10} D_{x}+a_{01} D_{y}+a_{00}$, where $r_{1}, a_{10}, a_{01}, a_{00} \in K$. The only non-trivial solution is

$$
a_{10}=a_{00}=0, r_{1}=-a_{01}, a_{01}=f_{1}(y) \cdot Q,
$$

where $Q=e^{\int(b-r) d x}$ and $f_{1}(y) \in K$ is a parameter, while

$$
c=0
$$

is a necessary condition of the solution's existence. Therefore, every family of the type $(X)(X Y)$ is parameterized by one function of one parameter (can be a constant function). Secondly, the initial factorization has the form

$$
L=\left(D_{x}+r\right) \circ\left(D_{x}+b\right) \circ D_{y},
$$

that is the operator $L$ itself has very special form.

Now, if we consider a factorization of the family in general form, namely

$$
\widetilde{L}_{1} \circ \widetilde{L}_{2}=\left(D_{x}+\widetilde{r}\right) \circ\left(D_{x y}+\widetilde{a} D_{x}+\widetilde{b} D_{y}+\widetilde{c}\right)
$$

where all the coefficients belong to $K$, and equates the corresponding product to the expression (5.1), we obtain

$$
\widetilde{a}=\widetilde{c}=0,
$$


and so any factorization of the family has the form

$$
L=\left(D_{x}+\widetilde{r}\right) \circ\left(D_{x}+\widetilde{b}\right) \circ D_{y} .
$$

Therefore, only reducible families of factorizations into two factors may exist in this case. Then, by Remark 2.4 there is no any irreducible family of factorizations into any number of factors in this case.

Consider the case in which all the factors of the symbol $\mathrm{Sym}_{L}$ are the same (up to a multiplicative function from $K$ ). Then one can find variables in which the symbol is $X^{3}$. Note that any irreducible factorization of the factorization type $(X)(X)(X)$ is an irreducible factorization of the types $(X)\left(X^{2}\right)$ and $\left(X^{2}\right)(X)$ also. Then because of the symmetry only one of two types $(X)\left(X^{2}\right)$ and $\left(X^{2}\right)(X)$ has to be considered. Therefore, it is sufficient to consider the factorization type $(X)\left(X^{2}\right)$. Thus, consider an initial factorization of the form

$$
L=L_{1} \circ L_{2}=\left(D_{x}+r\right) \circ\left(D_{x x}+a D_{x}+b D_{y}+c\right)
$$

where $r, a, b, c \in K$. Under the gauge transformations we may assume $a=0$ (while the coefficient at $D_{x}$ in the second factor of other factorizations of the family may be still non-zero). Consider the linearized problem (3.1) for such the initial factorization: the equation $F_{1} \circ L_{2}+L_{1} \circ F_{2}=0$ w.r.t. $F_{1}=r_{1}, F_{2}=$ $a_{10} D_{x}+a_{01} D_{y}+a_{00}$, where $r_{1}, a_{10}, a_{01}, a_{00} \in K$. The only non-trivial solution is $a_{01}=0, r_{1}=-a_{10}, a_{00}=-r a_{10}-\partial_{x}\left(a_{10}\right)$, provided both

$$
b=0
$$

and $c a_{10}+r^{2} a_{10}+2 r \partial_{x}\left(a_{10}\right)+a_{10} \partial_{x}(r)+\partial_{x x}\left(a_{10}\right)=0$. The solution of the latter equation depends on two arbitrary function in the variable $y$. Therefore, any family of the type $(X)(X X)$ is parameterized by two functions of one variable (can be constant functions), and such a family may exist only for an almost ordinary operator $L$. This implies that a family of the factorization type $(X)(X)(X)$ may exist only for an almost ordinary operator $L$.

Any irreducible family of the type $(X)(X)(X)$ serves as an irreducible family of the type $(X)(X X)$. Therefore, a family of the type $(X)(X)(X)$ can have two parameters (functions in one variables), that appear in the corresponding family of the type $(X)(X X)$, and additional parameters, that can appear when we consider two last factors separately. By the theorem 4.1 there is at most one additional parameter (a function in one variable). Thus, for the family of the type $(X)(X)(X)$ the maximal number of parameters is three, and these parameters are functions in one variable (may be constant functions). This agrees with [17.

The theorem implies that for an operator (with the completely factorable symbol), that is not almost ordinary, only reducible families may exist. Any such family is obtained by the multiplication (on the left or on the right) of a secondorder family by some non-parametric first order operator. Note that this secondorder family should be almost ordinary, by Theorem 4.1

Example 5.2. The family of the Landau operator (1.3) is reducible, which is obtained from a second-order family. 


\section{Fourth-Order Operators}

Here we start with an example of a fourth-order irreducible family for an almost ordinary operator.

Example 6.1. The following is a fourth-order irreducible family of factorizations:

$$
D_{x x x x}=\left(D_{x x}+\frac{2}{x+2 f_{1}(y)}+y\right)\left(D_{x x}-\frac{2}{x+2 f_{1}(y)}+y\right),
$$

where $f_{1}(y) \in K$ is a parameter.

Unlike the irreducible families of orders two and three, an irreducible fourthorder family need not be almost ordinary.

Example 6.2. The following is a fourth-order irreducible family of factorizations:

$D_{x x y y}=\left(D_{x}+\frac{\alpha}{y+\alpha x+\beta}\right)\left(D_{y}+\frac{1}{y+\alpha x+\beta}\right)\left(D_{x y}-\frac{1}{y+\alpha x+\beta}\left(D_{x}+\alpha D_{y}\right)\right)$, where $\alpha, \beta \in K \backslash\{0\}$. Note that the first two factors commute.

Again, we actually have several examples here. Namely, for the same operator $D_{x x y y}$, we have families of the types $(X)\left(X Y^{2}\right),(X Y)(X Y)$ and $(X)(Y)(X Y)$.

Theorem 6.3. In $K\left[D_{x}, D_{y}\right]$, irreducible fourth order families of factorizations with a completely factorable symbol exist only if in some system of coordinates their factorization types are $(X Y)(X Y),(X)\left(X Y^{2}\right),(X)(Y)(X Y)$ or $\left(X^{2}\right)\left(X^{2}\right)$, and symmetric to them.

Proof. For the symbol Sym of the family, there are exactly four possibilities: to have exactly four $\left(\mathrm{Sym}=S_{1} S_{2} S_{3} S_{4}\right)$, three $\left(\mathrm{Sym}=S_{1}^{2} S_{2} S_{3}\right)$, two $\left(\mathrm{Sym}=S_{1}^{2} S_{2}^{2}\right.$ and Sym $\left.=S_{1} S_{2}^{3}\right)$, or no $\left(\right.$ Sym $\left.=S_{1}^{4}\right)$ different factors.

Consider factorizations into two factors first. Because of properties of factorizations of adjoint operators (see in more detail in the proof of Theorem 5.1), it is enough to consider factorization type $\left(S_{i}\right)\left(S_{j}\right)$ instead of consideration of both $\left(S_{i}\right)\left(S_{j}\right),\left(S_{j}\right)\left(S_{i}\right)$. Also, recall that by Theorem 2.5 , no family of type $\left(S_{1}\right)\left(S_{2}\right)$, where $S_{1}, S_{2}$ are coprime exists. Thus, it is enough to consider the following cases:

I. $\operatorname{Sym}=S_{1}^{2} S_{2} S_{3}$.

1) $\left(S_{1} S_{2}\right)\left(S_{1} S_{3}\right)$

2) $\left(S_{1}\right)\left(S_{1} S_{2} S_{3}\right)$.

II. $\mathrm{Sym}=S_{1}^{2} S_{2}^{2}$.

1) $\left(S_{1} S_{2}\right)\left(S_{1} S_{2}\right)$.

2) $\left(S_{1}\right)\left(S_{1} S_{2}^{2}\right)$.

III. Sym $=S_{1} S_{2}^{3}$.

1) $\left(S_{1} S_{2}\right)\left(S_{2}^{2}\right)$.

3) $\left(S_{2}\right)\left(S_{1} S_{2}^{2}\right)$.

IV. Sym $=S_{1}^{4}$.

1) $\left(S_{1}\right)\left(S_{1}^{3}\right)$.

3) $\left(S_{1}^{2}\right)\left(S_{1}^{2}\right)$. 
$I$. Case Sym $=S_{1}^{2} S_{2} S_{3}$. In this case, in appropriate variables, the symbol has the form $\operatorname{Sym}_{L}=X^{2} Y(\alpha X+Y)$, where $\alpha \in K \backslash\{0\}$.

1) Case of the type $(X Y)(X(\alpha X+Y))$. We prove that there is no irreducible family of this type. Let $L_{1} \circ L_{2}=\left(D_{x y}+a_{1} D_{x}+b_{1} D_{y}+c_{1}\right) \circ\left(\alpha D_{x x}+D_{x y}+a_{2} D_{x}+\right.$ $b_{2} D_{y}+c_{2}$ ), where all coefficients are in $K$, be the initial factorization of such a family. Then under the gauge transformation we may assume $b_{2}=0$ (while the coefficient at $D_{y}$ in the second factor of any other factorization of the family may be still non-zero). Consider the linearized problem: the equation $F_{1} \circ L_{2}+L_{1} \circ F_{2}=0$ w.r.t. $F_{1}, F_{2} \in K[D]$ and $\operatorname{ord}\left(F_{1}\right)=\operatorname{ord}\left(F_{2}\right)=1$. The only non-trivial solution is parameterized by a function $f_{1}(y) \in K$ and exists only under two conditions on the coefficients of $L_{1}$ and $L_{2}: c_{1}=\partial_{y}\left(b_{1}\right)+b_{1} a_{1}$ and $c_{2}=\partial_{x x}(\alpha)-\partial_{x}\left(a_{2}\right)$. Now we come back to the initial problem and look for a family of factorizations in the general form: $L_{1} \circ L_{2}=\left(L_{1}+S_{1}\right) \circ\left(L_{2}+S_{2}\right)$, where $S_{1}, S_{2}$ are arbitrary first-order operators in $K[D]$. This gives us a system of equations in the coefficients of $S_{1}$ and $S_{2}$. The system together with conditions $c_{1}=\partial_{y}\left(b_{1}\right)+b_{1} a_{1}$ and $c_{2}=\partial_{x x}(\alpha)-\partial_{x}\left(a_{2}\right)$ has a unique non-trivial solution. The corresponding (to this solution) family of factorizations is complete, that is, both factors are factorable themselves: $L_{1} \circ L_{2}=$ $\left(D_{y}+a_{1}\right) \circ\left(D_{x}+b_{1}+\frac{Q}{W+f_{1}(y)}\right) \circ\left(D_{x}-\frac{Q}{W+f_{1}(y)}\right) \circ\left(\alpha D_{x}+D_{y}+a_{2}-\partial_{x}(\alpha)\right)$, where $Q=e^{-\int b_{1} d x}$ and $W=\int Q d x$ and $f_{1}(y)$ is the only parameter function. Now it is clear that the first and the last factors do not depend on a parameter, and so any factorization of any family of the type $(X Y)(X(\alpha X+Y))$ is reducible.

2 ) Case of the type $\left(S_{1}\right)\left(S_{1} S_{2} S_{3}\right)$. We prove now that there is no irreducible family of this type. By a change of variables one can make $S_{1}=X, S_{2}=Y$, $S_{3}=\alpha X+Y$, where $\alpha \in K$. Then consider a factorization of type $\left(S_{1}\right)\left(S_{1} S_{2} S_{3}\right)$,

$$
L_{1} \circ L_{2}=\left(D_{x}+c_{1}\right) \circ\left(D_{x x y}+D_{x y y}+a D_{x x}+b D_{x y}+c D_{y y}+d D_{x}+e D_{y}+f\right) \text {, }
$$

where all the coefficients belong to $K$, as the initial factorization of a family of factorizations. Under the gauge transformations we may assume $c=0$ (note that the analogous coefficients in the other factorizations of the family do not necessary become zero). Proceeding as in the previous case, we also get that there is only one non-trivial solution, which is parameterized by a function $f_{1}(y) \in K$. Also we have two conditions which provide the existence of such an equation: $e_{2}=$ $b_{2} c_{2}+\partial_{x}\left(b_{2}\right)-\alpha \partial_{x}\left(c_{2}\right)-\alpha c_{2}^{2}-2 \partial_{x}(\alpha) c_{2}-\partial_{x x}(\alpha), f_{2}=d_{2} c_{2}+\partial_{x}\left(d_{2}\right)-a_{2} \partial_{x}\left(c_{2}\right)-$ $a_{2} c_{2}^{2}-2 \partial_{x}\left(a_{2}\right) c_{2}-\partial_{x x}\left(a_{2}\right)$. Now, we use the obtained conditions for the initial problem, where a family of factorizations is considered in general form. Thus, we get that if such a family exists, then the second factor of the family can be always factored into first and second-order operators, and the second-order operator does not depend on the parameter: $L_{1} \circ L_{2}=\left(D_{x}+c_{1}+m_{00}\right) \circ\left(D_{x}+c_{2}-m_{00}\right) \circ$ $\left(\alpha D_{x}+D_{y}+a_{2} D_{x}+\left(b_{2}-\alpha c_{2}-\partial_{x}(\alpha)\right) D_{y}+d_{2}-a_{2} c_{2}-\partial_{x}\left(a_{2}\right)\right)$, where only $m_{00} \in K$ may depend on a parameter. Thus any family (if it exists) of the type $(X)(X Y(\alpha X+Y))$ is reducible.

$I I$. Case of symbol Sym $=S_{1}^{2} S_{2}^{2}$. 
1) Case of the type $\left(S_{1} S_{2}\right)\left(S_{1} S_{2}\right)$. A family exists. See example (6.2).

2) Case of the type $\left(S_{1}\right)\left(S_{1} S_{2}^{2}\right)$. A family exists. See example (6.2).

III. Case of symbol Sym $=S_{1} S_{2}^{3}$.

1) $\left(S_{1} S_{2}\right)\left(S_{2}^{2}\right)$. In this case, in appropriate variables, the symbol has form $(X Y)\left(Y^{2}\right)$. We prove that there is no irreducible family of this type. Indeed, consider a factorization of the considering type: $L_{1} \circ L_{2}=\left(D_{x y}+a_{1} D_{x}+b_{1} D_{y}+c_{1}\right) \circ$ $\left(D_{y y}+a_{2} D_{x}+b_{2} D_{y}+c_{2}\right)$ with all coefficients in $K$, as the initial factorization of a family of factorizations. By the gauge transformations we may assume $c_{2}=0$. The linearized problem is the equation $F_{1} \circ L_{2}+L_{1} \circ F_{2}=0$ w.r.t. $F_{1}, F_{2} \in K[D]$ and $\operatorname{ord}\left(F_{1}\right)=1, \operatorname{ord}\left(F_{2}\right)=1$. The equation has a non-trivial solution, which depends on two parameter functions $f_{1}(x), f_{2}(x) \in K$, and the existence is provided by the conditions $a_{2}=0, c_{1}=b_{1} a_{1}+\partial_{x}\left(a_{1}\right)$. Thus, a family may exist only if the considered operator $L$ has the form $L=\left(D_{x y}+a_{1} D_{x}+b_{1} D_{y}+b_{1} a_{1}+\partial_{x}\left(a_{1}\right)\right) \circ\left(D_{y}+b_{2}\right) \circ D_{y}$ for some $a_{1}, b_{1}, b_{2} \in K$. Then, one may prove that in this case any family of factorization has the form $L=\left(D_{x y}+\ldots\right) \circ\left(D_{y}+\ldots\right) \circ D_{y}$, meaning that is there is no irreducible fourth-order family of the type $(X Y)\left(Y^{2}\right)$.

2 ) Case of type $\left(S_{2}\right)\left(S_{1} S_{2}^{2}\right)$. In this case, in appropriate variables, the symbol has form $(Y)\left(X Y^{2}\right)$. We prove that there is no irreducible family of this type. Indeed, consider a factorization of this type: $L_{1} \circ L_{2}=\left(D_{y}+c_{1}\right) \circ\left(D_{x y y}+a D_{x x}+\right.$ $\left.b D_{x y}+c D_{y y}+d D_{x}+e_{1} D_{y}+f\right)$, with all coefficients in $K$, as the initial factorization of a family of factorizations. By gauge transformations we may assume the coefficient at $D_{y y}$ in this initial factorization is zero, that is $c=0$. The linearized problem is the equation $F_{1} \circ L_{2}+L_{1} \circ F_{2}=0$ w.r.t. $F_{1}, F_{2} \in K[D]$ and $\operatorname{ord}\left(F_{1}\right)=0, \operatorname{ord}\left(F_{2}\right)=2$. This equation has a non-trivial solution, provided $a=0$ and $d=e_{1}^{-2}\left(b f e_{1}-b e_{1} \partial_{y}\left(e_{1}\right)+e_{1}^{2} \partial_{y}(b)+3 f \partial_{y}\left(e_{1}\right)-e_{1} \partial_{y}(f)-2\left(\partial_{y}\left(e_{1}\right)\right)^{2}+\right.$ $\left.e_{1} \partial_{y y}\left(e_{1}\right)-f^{2}\right)$. Then, when we consider the corresponding family of factorizations in general form, we may apply these conditions, and easily get that such a family cannot exist.

$I V$. Case of no different factors of the symbol. Then there exist variables such that the symbol is $X^{4}$. Consider factorizations into two factors. Then, by Theorem 2.5 and because of the symmetry, it is enough to consider types of factorizations $(X)\left(X^{3}\right)$ and $\left(X^{2}\right)\left(X^{2}\right)$.

1) Case of the type $(X)\left(X^{3}\right)$. Prove that there is no irreducible family of the type $(X)\left(X^{3}\right)$. Consider a factorization of the type $(X)\left(X^{3}\right): L_{1} \circ L_{2}=\left(D_{x}+\right.$ $\left.c_{1}\right) \circ\left(D_{x x x}+a D_{x x}+b D_{x y}+c D_{y y}+d D_{x}+e D_{y}+f\right)$, where all coefficients are in $K$. Solving the linearized problem, we get that a family in this case may be parameterized by only one function in one variable, and such a family may exist provided two conditions on the initial coefficients hold (one of them is just $c=0$ ). Then, when we look for a family in general form, one may prove that such families indeed can exist, but all such families are reducible.

2) Case of the type $\left(X^{2}\right)\left(X^{2}\right)$. Here we have the Example 6.1 of a family of factorizations depended on one functional parameter in one variable. In fact the maximal number of parameters in this type of factorization is four [17. 
Now consider factorizations into more than two factors. By Remark 2.4 and symmetry properties, irreducible families into four and three factors cannot exist in cases $I, I I I$ and $I V$. Consider parametric factorizations into three factors in case $I I$, Sym $=S_{1}^{2} S_{2}^{2}$. It is enough to consider the following cases:

a. $\left(S_{1}\right)\left(S_{2}\right)\left(S_{1} S_{2}\right)$.

b. $\left(S_{1}\right)\left(S_{1} S_{2}\right)\left(S_{2}\right)$.

a. A family exists. See example 6.2.

$b$. Prove that there is no irreducible family of type $(X)(X Y)(Y)$ (in appropriate variables, $S_{1}=X$ and $\left.S_{2}=Y\right)$. Consider an initial factorization of this factorization type: $L=L_{1} \circ L_{2} \circ L_{3}=\left(D_{x}+c_{1}\right)\left(D_{x y}+a D_{x}+b D_{y}+c\right)\left(D_{y}+c_{3}\right)$, where all the coefficients belong to $K$, and are known functions. Solving the linearized problem, i.e. (3.2), we get $c=a_{x}+a b$. This means that $L_{2}=\left(D_{x}+b\right) \circ\left(D_{y}+a\right)$. As every factorization of a family can be chosen as the initial, then the second factor is factorable for every factorization of the family.

Suppose there is another factorization of the same operator of the same factorization type, then it has form $L=M_{1} \circ M_{2} \circ M_{3} \circ M_{4}=\left(D_{x}+m_{1}\right)\left(D_{x}+\right.$ $\left.m_{01}\right)\left(D_{y}+m_{01}\right)\left(D_{y}+m_{3}\right)$, where all the coefficients belong to $K$, and are some unknown functions. Equate the corresponding coefficients of these two factorizations of $L$. Then, one can easily find expressions for $m_{1}, m_{01}, m_{01}, m_{3}$ in terms of $a, b, c_{1}, c_{3}$ and two parameter functions $F_{1}(x)$ and $F_{2}(y)$. However, if we compute the composition of $M_{1}$ and $M_{2}$, all the parameters disappear, and, therefore, any parametric family of factorization type $\left(S_{1}\right)\left(S_{1} S_{2}\right)\left(S_{2}\right)$ is reducible.

Cases $a$. and $b$. also imply that irreducible families into four factors cannot exist in case $I I$.

\section{Conclusion}

For second, third and fourth order LPDOs with completely factorable symbols on the plane, we have completely investigated what factorizations' types admit irreducible parametric factorizations. For these factorization types, examples are given. Note the our method is general and we cover the case of the ordinary operators as a particular case. For operators of orders two and three, we describe in addition the structure of their families of factorizations. For the partial operators of order four, the question remains open (for ordinary operators the possible number of parameters in a family of factorizations has been investigated in [17). For the case of partial differential operators we would surmise that no more than two or one parameters (which could be functions) are possible. Generalizations to LPDOs with arbitrary symbols (without the complete factorization assumption), to high order LPDOs, and to those in multiple-dimensional space are of interest also. 


\section{References}

[1] I. Anderson, M. Juras, Generalized Laplace Invariants and the Method of Darboux. Duke J. Math., 89(1997), 351-375.

[2] I. Anderson, N. Kamran, The Variational Bicomplex for Hyperbolic Second-Order Scalar Partial Differential Equations in the Plane. Duke J. Math., 87(1997), 265-319.

[3] C. Athorne. A $Z \times R$ Toda system. Phys.Lett.A., 206(1995), 162-166.

[4] H.Yilmaz, C.Athorne. The geometrically invariant form of evolution equations. J.Phys.A., 35(2002), 2619-2625.

[5] D.Grigoriev, F.Schwarz. Factoring and Solving Linear Partial Differential Equations., J.Computing, 73(2004), 179-197.

[6] D.Grigoriev, F.Schwarz. Generalized Loewy-Decomposition of D-Modules. Proc. ISSAC2005, ACMPress, 2005, 163-170.

[7] G. Darboux, Théorie Générale des Surfaces, Chelsea, New York, 1972.

[8] U. Dini. Sopra una classe di equazioni a derivate parziali di second ordine con un numero qualunque di variabili. Atti Acc. Naz. dei Lincei. Mem. Classe fis., mat., nat. (ser. 5) 4(1901), 121-178. Also Opere III(1901), 489-566.

[9] U. Dini. Sopra una classe di equazioni a derivate parziali di secondordine. Atti Acc. Naz. dei Lincei. Mem. Classe fis., mat., nat. (ser. 5), 4(1902), 431-467. Also Opere III(1902), 613-660.

[10] M. Juras. Generalized Laplace invariants and classical integration methods for second order scalar hyperbolic partial differential equations in the plane. Proc. Conf. Diff. geometry and applications, Brno, Czech Republic (1996), 275-284.

[11] Z. Li, F. Schwarz, S.P. Tsarev. Factoring systems of linear PDEs with finitedimensional solution spaces. J. Symbolic Computation, 36(2003), 443-471.

[12] J. Le Roux. Extensions de la méthode de Laplace aux 'equations lin'eaires aux derivées partielles dórdre sup'erieur au second. Bull. Soc. Math. de France, 27(1899), 237-262. A digitized copy is obtainable from http://www.numdam.org/

[13] E. Shemyakova, F. Winkler, Obstacle to Factorization of LPDOs, Proc. Transgressive Computing, Granada, Spain, 2006.

[14] E. Shemyakova, F. Winkler, Obstacles to the Factorization of Linear Partial Differential Operators into Several Factors., J. Programming and Computer Software (2006), accepted.

[15] V.V. Sokolov and A.V. Zhiber. On the Darboux integrable hyperbolic equations. Physics Letters A, 208(1995), 303-308.

[16] S.P. Tsarev. An algorithm for complete enumeration of all factorizations of a linear ordinary differential operator. Proceedings of ISSAC96, ACM Press, 226-231.

[17] S.P. Tsarev. Factorization of linear partial differential operators and Darboux integrability of nonlinear PDEs. SIGSAM Bulletin, 32(1998), No. 4., 21-28. Also Computer Science e-print cs.SC/9811002 at http://www.arxiv.org/

[18] S.P. Tsarev. Generalized Laplace Transformations and Integration of Hyperbolic Systems of Linear Partial Differential Equations. Proc. ISSAC'05, 325-331.

[19] S.P. Tsarev. On factorization and solution of multidimensional linear partial differential equations. Computer Science e-print cs.SC/0609075 at http://www.arxiv.org/ 2006. 


\section{Acknowledgment}

This work was supported by Austrian Science Foundation (FWF) under the project SFB F013/F1304.

Ekaterina Shemyakova

Research Institute for Symbolic Computations (RISC)

Johannes Kepler University

Altenbergerstr. 69

A-4040 Linz

Austria

e-mail: kath@risc.uni-linz.ac.at 